University of New Hampshire

University of New Hampshire Scholars' Repository

Space Science Center

Institute for the Study of Earth, Oceans, and

Space (EOS)

1994

\title{
An overview of solar flare results from COMPTEL
}

Mark L. McConnell

University of New Hampshire - Main Campus, mark.mcconnell@unh.edu

Follow this and additional works at: https://scholars.unh.edu/ssc

Part of the Astrophysics and Astronomy Commons

\section{Recommended Citation}

An overview of solar flare results from COMPTEL McConnell, Mark, AIP Conference Proceedings, 294, 21-25 (1994), DOl:http://dx.doi.org/10.1063/1.45193

This Conference Proceeding is brought to you for free and open access by the Institute for the Study of Earth, Oceans, and Space (EOS) at University of New Hampshire Scholars' Repository. It has been accepted for inclusion in Space Science Center by an authorized administrator of University of New Hampshire Scholars' Repository. For more information, please contact Scholarly.Communication@unh.edu. 


\section{AIP $\mid$ Proceedings}

\section{An overview of solar flare results from COMPTEL}

Mark McConnell

Citation: AIP Conference Proceedings 294, 21 (1994); doi: 10.1063/1.45193

View online: http://dx.doi.org/10.1063/1.45193

View Table of Contents:

http://scitation.aip.org/content/aip/proceeding/aipcp/294?ver=pdfcov

Published by the AIP Publishing

Articles you may be interested in

OSSE observations of the 4 June 1991 solar flare

AIP Conf. Proc. 294, 15 (1994); 10.1063/1.45214

Observations of the 1991 June 11 solar flare with COMPTEL

AIP Conf. Proc. 294, 100 (1994); 10.1063/1.45207

COMPTEL observations of gammaray flares in October 1991

AIP Conf. Proc. 294, 55 (1994); 10.1063/1.45200

COMPTEL's solar flare catalog

AIP Conf. Proc. 294, 51 (1994); 10.1063/1.45199

Xray and gammaray observations of solar flares by GRANAT AIP Conf. Proc. 294, 3 (1994); 10.1063/1.45197 


\title{
AN OVERVIEW OF SOLAR FLARE RESULTS FROM COMPTEL
}

\author{
Mark McConnell \\ Space Science Center, University of New Hampshire, \\ Durham, NH 03824
}

\begin{abstract}
The COMPTEL experiment on the Compton Gamma Ray Observatory (CGRO) has been operating in orbit since April of 1991. During that time, COMPTEL has observed several large flares, the most notable of which were several X-class flares which took place in June of 1991. As a solar instrument, COMPTEL has the capability to measure solar flare radiation in two parallel observing modes. In its telescope mode, COMPTEL is capable of measuring both solar flare photons (in the $0.75-30 \mathrm{MeV}$ range) and solar flare neutrons (in the $20-150 \mathrm{MeV}$ range) using the double scatter technique with a field-of-view of $\sim 1$ steradian. This approach also permits the imaging of the incident solar radiations (both photons and neutrons). The burst mode of COMPTEL utilizes two of the lower D2 detectors as large-area spectroscopy detectors to provide additional data in the $0.6-10 \mathrm{MeV}$ range. Here we shall review both modes of COMPTEL operation and provide an overview of solar flare results which are presently available.
\end{abstract}

\section{INSTRUMENT DESCRIPTION}

The design of COMPTEL is illustrated schematically in Figure 1. COMPTEL has two modes of measuring $\gamma$-rays ${ }^{1}$. These two modes operate in parallel. The primary mode of operation is the double-scatter (or telescope) mode which is used for imaging incident radiations within a field-of-view of $\sim 1$ steradian. The burst mode utilizes two large area $\mathrm{NaI}$ detectors to provide additional spectroscopic data.

In the telescope mode an incoming $\gamma$-ray scatters off of an electron in one of seven D1 detectors and proceeds down to interact in one of fourteen D2 detectors. Each D1 detector is $28 \mathrm{~cm}$ in diameter by $8.5 \mathrm{~cm}$ thick and filled with liquid organic scintillator (NE213A). The low density and low $Z$ of Ne213A maximizes the probability of a single photon scatter followed by the subsequent escape of the scattered photon. Each D2 detector is composed of $\mathrm{NaI}$ (Tl) $28 \mathrm{~cm}$ in diameter by 7.5 $\mathrm{cm}$ thick. Events which scatter only once in D1 and are fully absorbed in D2 constitute an ideal telescope events. The photon scattering events in D1 take place according to the Compton kinematic formula

$$
\phi=\cos ^{-1}\left\{\left(1-\varepsilon / E_{2}+\varepsilon /\left(E_{1}+E_{2}\right)\right)\right\},
$$

where $\varepsilon$ is the electron rest mass energy, $\mathrm{E}_{1}$ is the energy deposit in D1, $\mathrm{E}_{2}$ is the energy deposit in $D_{2}$ and $\phi$ is the Compton scatter angle provided $E_{1}+E_{2}$ is the full incident $\gamma$-ray energy. 
As an imaging telescope, COMPTEL relies on the full energy deposit of the scattered $\gamma$-ray to correctly estimate the scattering angle $\phi$. For solar flares, we can select only those events where the inferred scatter angle $\phi$ about the vector of the scattered $\gamma$-ray is consistent with the solar direction. These events correspond to those which deposit the full energy of their scattered photon in D2. This type of event selection has the advantage that the the response of the telescope becomes Gaussian in shape with a heavily suppressed Compton tail at low energies. Since the solar $\gamma$ ray spectra are rich in nuclear lines, a simple response function facilitates

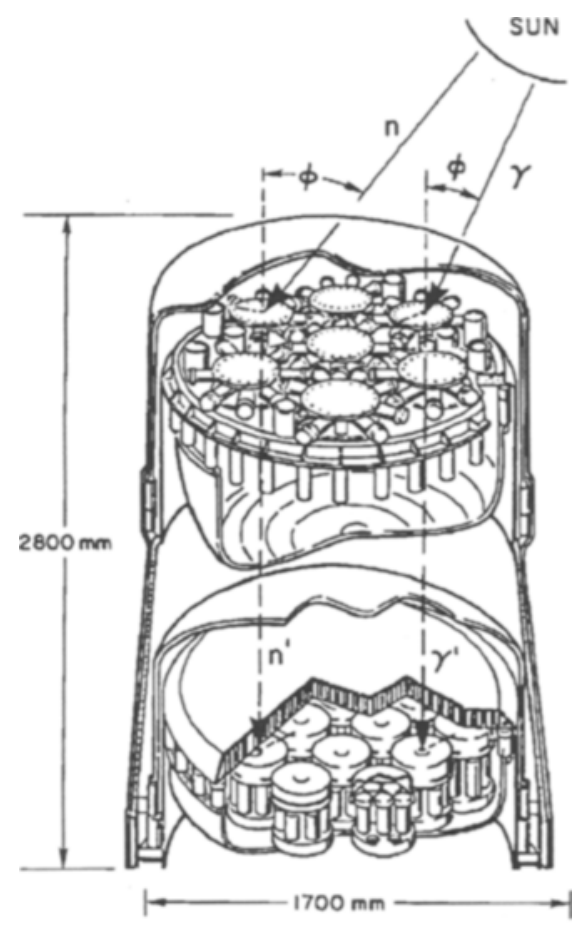

\section{COMPTEL}

\section{IMAGING COMPTON TELESCOPE}

Fig. 1. Schematic of COMPTEL with typical g-ray and neutron interactions. the de-convolution of the energy-loss spectra.

The D1 and D2 subsystems of the telescope are each completely surrounded by charged particle detectors. Four domes of plastic scintillator (NE110) are $1.5 \mathrm{~cm}$ thick and do not significantly attenuate the incident energetic $\gamma$-ray or neutron fluxes, yet they are virtually $100 \%$ efficient in identifying charged cosmic rays. The charged particle shields and other intervening material heavily attenuate the solar flare hard X-ray flux, minimizing pulse pile-up effects in the D1 and D2 detectors. They do, however, saturate under the intense soft thermal X-ray flux associated with flares and, for intense flares, may become a significant source of deadtime.

Since neutrons (like photons) are not detected by the charged particle veto domes, neutrons can produce photon-like events via elastic proton scattering in D1. COMPTEL can distinguish between photons and neutrons in two ways. First, the liquid scintillator in D1 possesses pulse shape discrimination (PSD) properties. This capability allows for efficient identification of signals from recoil protons produced by the elastic neutron scatters. Secondly, time-of-flight (TOF) measurements of the scattered particle traveling between D1 and D2 permit the identification of scattered neutrons.

The ideal type of neutron interaction in COMPTEL occurs when the incoming neutron scatters elastically off a hydrogen nucleus in the D1 detector. The scattered neutron then proceeds to the D2 detector where it interacts, depositing some of its 
energy to produce a trigger signal. The energy of the incident neutron is computed by summing the proton recoil energy $E_{1}$ in the D1 detector with the energy of the scattered neutron $E_{S}$ deduced from the TOF measurement. The scatter angle for nonrelativistic neutrons $(<150 \mathrm{MeV})$ can be computed by the formula:

$$
\tan ^{2} \phi=\mathrm{E}_{1} / \mathrm{E}_{\mathrm{s}} \text {. }
$$

As with $\gamma$-rays, neutrons can be traced backwards from D2 to D1 through the angle $\phi$ to a cone mantle restricting the incident direction to include the Sun. The pulse shape from recoil protons is sufficiently different from that of electrons to reject more than $95 \%$ of electron-recoil events greater than about $1 \mathrm{MeV}$, the energy threshold in D1 for neutron detection. The PSD and TOF criteria are normally set such that solar neutrons incident on D1 in the energy range from about $10 \mathrm{MeV}$ to $150 \mathrm{MeV}$ are recorded. In this energy interval COMPTEL can observe neutrons from about 14.5 to 55 minutes after release from the Sun.

The burst mode of the COMPTEL instrument can also be used to detect solar $\gamma$ rays $^{2}$. One D2 detector module (the low-range module) covers the energy interval from 0.1 to $1 \mathrm{MeV}$ and another (the high-range module) covers the interval from $\sim 1$ to $10 \mathrm{MeV}$. Each detector module has an unobscured field-of-view of about $2.5 \mathrm{sr}$ and a physical area of $\sim 600 \mathrm{~cm}^{2}$. Outside this field-of-view varying amounts of intervening material exist which attenuate the solar $\gamma$-ray flux. These detectors operate in parallel with the telescope data, continuously accumulating spectra with 100 second integration time. Upon receipt of a BATSE trigger, these detectors begin a series of shorter accumulations (from 0.1 to 6 seconds in duration) which permit a more careful study of the burst (or flare) event. During the time period from May of 1991 to May of 1992, the low range detector of COMPTEL was turned off due to an intermittent PMT (one of seven PMTs on each D2 module). This PMT has now failed completely, allowing us to make full use of this module for burst and solar flare studies.

\section{OBSERVATIONS}

Since the time of the GRO launch (on 5 April 1991), the most notable period of solar activity was in June of 1991 with the appearance of active region 6659. From the emergence of this region on the east limb on 1 June until 8 June the Sun was outside the field-of-view of COMPTEL. The occurence of several major flares during this time prompted the declaration of a CGRO Target of Opportunity. From 8 June until passage of the region around the west limb on 15 June, CGRO was reoriented so that the Sun remained within the COMPTEL field-of-view (at a zenith angle of $\sim 10^{\circ}$ to $15^{\circ}$ ). Major (X-class) flares were recorded on 9,11 and 15 June 1991. Intial results from these flares have already been reported $3-6$. A more detailed discussion of the June 9 flare is given elsewhere in these proceedings 7 . Here we shall provide a few typical examples of COMPTEL solar flare data.

Consider first, the flare of June 11. This flare took place within the COMPTEL FoV shortly after satellite sunrise. The impulsive phase (according to the GOES Xray data) extended from 0158 UT until approximately $0210 \mathrm{UT}$, after which there was emission lasting until at least 0225 UT. Figure 2 shows a time history of this flare as observed by the COMPTEL high-range burst module (0.6-10 MeV). A telescopemode energy-loss spectrum from the impulsive phase of the flare is shown in Figure 
3. Here we see evidence for the strong $2.2 \mathrm{MeV}$ line emission which is also measured by COMPTEL in each of the two orbits following the impulsive phase ${ }^{6}$. This observation of extended emission is especially interesting given the observation by EGRET of emission at energies $>50 \mathrm{MeV}$ lasting for up to 10 hours after the impulsive phase ${ }^{8}$.

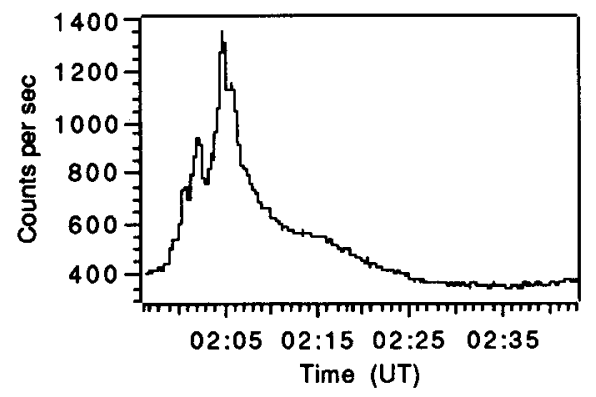

Fig. 2. Time history of the June 11 flare as measured by the high-range burst module $(0.6-10 \mathrm{MeV})$

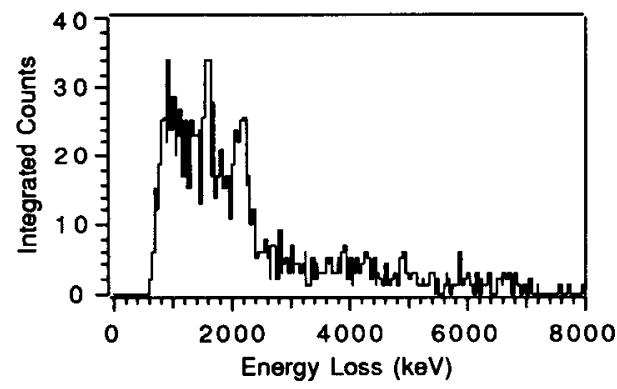

Fig. 3. The energy loss spectrum from the impulsive phase of the June 11 flare.
Extended emission (lasting on the order of hours after the impulsive phase) appears to have been a rather common feature of those flares associated with AR6659. Another example is the flare of June 15 . The impulsive phase of this $\mathrm{X} 12+$ flare, which peaked at $0820 \mathrm{UT}$, was unobserved by CGRO due to orbital darkness. It was not until some 40 minutes after the flare onset that COMPTEL was able to collect its first data from this event. This flare was also measured by the GAMMA-1 instrument ${ }^{9}$ and was reported to have emission $>30 \mathrm{MeV}$ after $\sim 0837$ UT lasting for at least 20 minutes after the $\mathrm{X}$-ray maximum. The COMPTEL measurements began at $\sim 0859 \mathrm{UT}$, while the GAMMA-1 measurements ended at 0902 UT. The COMPTEL instrument continued making measurements until 0930 UT. There is no apparent signature of this event in the burst mode data, a testament to the sensitivity of the telescope mode. The light curve as measured with the telescope mode data is shown in Figure 4. The e-folding decay time of this emission is measured to be $910 \pm 135$ seconds.

Figure 5 demonstrates the neutron capability of COMPTEL using data from the June 15 flare. The plot shows the neutron production time history. The data points are plotted at the arrival time of photons created simultaneously with the neutrons. The curve suffers from velocity dispersion effects, in that the counts at the earliest times in the plot are the slowest neutrons, while the counts at the later times come from higher energy neutrons. The time intervals do not sample uniformly the available neutron energy spectrum. The plot does show, however, that neutron emission occurred at times near the $\mathrm{X}$-ray maximum and extended for over an hour. This, coupled with the detection of $\gamma$-rays up to $0930 \mathrm{UT}$, means that proton acceleration or precipitation persisted for at least 70 minutes. 
Several other flares have been observed in the COMPTEL telescope mode. Many of these flares have not yet been studied in the same detail as the large flares of June 1991. An effort to catalog all of the telescope mode flare events is underway. A first report of this effort, concentrating on the flares of October 1991, can be found elsewhere in these proceedings 10 .

Similarly, a catalog is being compiled of all of those events detected by the COMPTEL burst mode detectors. This list will be considerably larger than the telescope mode catalog because the burst modules are sensitive to a much larger fraction of the sky. A status report on this effort can also be found in these proceedings ${ }^{11}$.

\section{CONCLUSIONS}

COMPTEL has proven itself as a very effective tool for the study of solar flare phenomena. The CGRO Target of Opportunity in June of 1991 has already provided some valuable results. Although most of the reported results todate have concentrated on the June flares, much effort remains in studying the other flare events in the COMPTEL database. Cataloging efforts are already underway, in preparation for a more careful study of all available events.

\section{REFERENCES}

1. V. Schönfelder et al., Ap. J. (Suppl.), ㅁ6, 629 (1993).

2. C. Winkler et al., Adv. Space Res. 6 113-117 (1986).

3. M. McConnell et al., Adv. Sp. Res., to be published (1992).

4. J. Ryan et al., Adv. Sp. Res., to be published (1992).

5. J. Ryan et al., Compton Centennial Symposium Proc., to be published (1993).

6. G. Rank et al., Compton Centennial Symposium Proc., to be published (1993).

7. J. Ryan et al., these proceedings.

8. G. Kanbach et al., Astr. Ap. Suppl., 27, 349 (1993).

9. V. V. Akimov et al., in Proceedings of the 22nd International Cosmic Ray Conference, (Dublin, Ireland, 1991), $\underline{3}, 73$.

10. M. Varendorff et al., these proceedings.

11. R. Suleiman et al., these proceedings. 